\title{
Safety and Efficacy of Laser Enucleation of the Prostate in Elderly Patients - A Narrative Review
}

\author{
Mehmet Yilmaz (D) \\ Julia Esser \\ Rodrigo Suarez-lbarrola \\ Christian Gratzke \\ Arkadiusz Miernik (D)
}

University of Freiburg - Medical Centre, Faculty of Medicine, Department of Urology, Freiburg, Germany
Correspondence: Arkadiusz Miernik University of Freiburg - Medical Centre, Faculty of Medicine, Department of Urology, Hugstetter Str. 55, Freiburg,

D-79106, Germany

$\mathrm{Tel}+4976 \mid 270-2582$ I

Fax +49 76I 270-25829

Email arkadiusz.miernik@uniklinik-

Freiburg.de

\begin{abstract}
Benign prostatic hyperplasia $(\mathrm{BPH})$ is a common health condition in aging men resulting in lower urinary tract symptoms (LUTS) and low quality of life (QoL). We aimed to review studies on laser enucleation of the prostate (LEP) due to LUTS in elderly patients ( $>65$ years) comparing different age groups, particularly considering functional outcomes, safety, and perioperative complications. A comprehensive search was conducted using the PubMed-MEDLINE, Web of Science, Wiley Online Library and Cochrane Library databases over the last 10 years until 7 October 2021 with the following search terms solely or in combination: "laser enucleation", "prostate", "laser prostatectomy", "aging", "elderly patients", "older patients" and "age". We identified 12 articles for inclusion in our analysis. Five studies focussed mainly on holmium laser enucleation of the prostate (HoLEP), one compared HoLEP to other laser prostatectomy procedures, two studies examined thulium laser enucleation of the prostate (ThuLEP), and two studies focused on GreenLight laser, one study focussed on potassium-titanyl-phosphate (KTP) laser, and another study compared laser prostatectomy to transurethral resection of the prostate (TURP). These studies showed that LEP improved IPSS, Qmax and QoL compared to baseline regardless of age; no significant difference was reported among age groups. Although ASA scores and anticoagulation/antiplatelet therapy rates are higher in elderly patients, studies revealed no significant difference in perioperative complication rates between age groups. Laser enucleation of the prostate is an effective and safe procedure in elderly patients, resulting in good functional outcomes, low morbidity, and few perioperative complications.
\end{abstract}

Keywords: aged, benign prostatic hyperplasia, elderly patients, HoLEP, laser enucleation of the prostate

\section{Introduction}

Benign prostatic hyperplasia $(\mathrm{BPH})$ is a common health condition in aging men resulting in lower urinary tract symptoms (LUTS) and low quality of life (QoL). ${ }^{1}$ Studies on BPH epidemiology from 25 countries show a combined prevalence of $26.2 \%$, with its prevalence rising with age. ${ }^{2}$ Transurethral resection of the prostate (TURP) and open prostatectomy (OP) have been considered for decades the surgical standard for treating BPH. ${ }^{3}$ Yet TURP and OP are associated with intraoperative and postoperative complications such as bleeding, clot retention requiring intervention, genitourinary infections, fluid absorption, and TUR syndrome. ${ }^{3-5}$ Therefore, it is possible to deteriorate the quality of life after these procedures, especially in the elderly and comorbid patients. Elderly patients may be taking anticoagulants for cardiovascular disease and suffer from comorbidities such as hypertension, renal insufficiency or diabetes mellitus; they therefore require procedures less invasive than TURP and OP. ${ }^{6}$ 
Laser enucleation of the prostate (LEP) is now a frequent and widespread alternative, minimally invasive approach to TURP and OP in the surgical treatment of $\mathrm{BPH}^{7,8}$ Holmium: YAG, Thulium, potassium-titanylphosphate (KTP), lithium triborate (LBO) laser, GreenLight and Diode laser are the laser types now used in surgically treating symptomatic $\mathrm{BPH}^{7}$ There is evidence that LEP is similarly safe and efficacious as TURP. ${ }^{7,9,10}$ In addition, LEP has also been shown to be associated with increased QoL. ${ }^{11,12}$ However, studies on LEP in elderly patients are scarce, and there is no collective evaluation study providing efficacy and safety results. We therefore saw the need to review the safety and efficacy of LEP according to age groups and in the elderly population. We aimed to discuss LEP due to LUTS in elderly patients ( $>65$ years) comparing different age groups, especially considering functional outcomes and safety.

\section{Materials and Methods}

\section{Literature Search}

A comprehensive search was conducted using the PubMed-MEDLINE, Web of Science, Wiley Online Library and Cochrane Library databases over the last 10 years until 7 October 2021 with the following search terms solely or in combination: "laser enucleation", "prostate", "laser prostatectomy", "aging", "elderly patients", "older patients" and "age". After retrieving the titles and abstracts of selected articles, the full texts of related articles were screened. The article selection process is shown in Figure 1. The PICOS: Population (P), Intervention (I), Comparison (C), Outcomes (O), and Study design (S) approach was used to determine eligibility criteria. ${ }^{13} \mathrm{We}$ thus selected studies in which BPH patients (P) underwent laser enucleation of the prostate and/or other laser prostatectomy procedures (I) specified various age groups were compared (including $>65$ years) (C), to assess functional outcomes, comorbidities, perioperative complications, and safety $(\mathrm{O})$ in prospective or retrospective studies (S).

We excluded studies unrelated to laser enucleation of the prostate, or without objectives or outcomes related to mainly age, those that did not perform age classification and/or failed to specify a specific age cut-off regarding surgical outcome, or were not written in English, as well as case reports, review articles, editorials, reply to authors, and conference abstracts.

\section{Data Extraction}

Articles relevant to our subject of interest were retrieved and evaluated independently by two authors (M.Y. and J. E.). Authors and date of study, study design, age stratifications, number of patients included, mean age, preoperative prostate volume (cc), total serum PSA $(\mathrm{ng} / \mathrm{mL})$, International Prostate Symptom Score (IPSS) (a questionnaire consisting of seven symptom questions and a global QoL question for LUTS diagnosis and treatment followup), maximum urinary flow rate (Qmax) (a basic noninvasive urodynamic test), quality of life (QoL) (a subjective concept determined by various surveys), length of hospital stay (LOS), operative time, American Society of Anesthesiology (ASA) scores (a classification system in which patients are evaluated according to the risk of anaesthesia before performing surgery), antiplatelet/anticoagulation (AP/AC) therapy status', comorbidities, complications according to the Clavien- Dindo classification (a system for grading the severity of surgical complications) were recorded. Descriptive statistics were used for basic data, and a narrative synthesis was created.

\section{Results}

A total of 389 articles were identified through our search query. Overall, 359 publications were excluded after reviewing the title and abstract for these reasons: not related to laser enucleation of the prostate $(\mathrm{n}=28)$, objective or outcome not related to mainly age $(n=222)$, not written in English $(n=16)$, case reports $(n=13)$, review articles $(n=33)$, conference abstracts $(n=31)$, editorials $(n=8)$, reply to the authors $(n=8)$. After full-text evaluation, we excluded 18 more articles that did not classify by age and/or failed to specify a specific age cut-off regarding surgical outcomes. The remaining 12 articles were ultimately incorporated in our review. Table 1 presents a summary of baseline characteristics, perioperative data, and the main findings of the studies included in our review. Eight of them were retrospective ${ }^{14-21}$ and 4 prospective. ${ }^{6,22-24}$ Five studies focussed mainly on Holmium laser enucleation of the prostate (HoLEP). ${ }^{6,17,20-22}$ One study focussed on comparing HoLEP to other laser prostatectomy procedures, ${ }^{16}$ two studies examined Thulium laser enucleation of the prostate (ThuLEP) in more detail, ${ }^{14,18}$ and two other studies focussed on the GreenLight XPS 180-W laser procedure, ${ }^{19,23}$ one focussed on potassium-titanyl-phosphate (KTP) $\operatorname{laser}^{24}$ and another compared laser prostatectomy to transurethral resection of the prostate (TURP). ${ }^{15}$ 


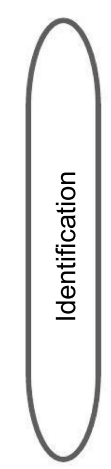

Records identified through PubMed-

Medline, Cochrane Library, Web of

Science and WileyOnline Library

$(n=389)$

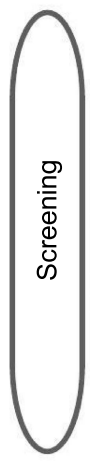

Articles screened

$(n=389)$

Records excluded after title and abstract review

$$
(n=359)
$$

(reviews, editorials, case reports, conference abstracts, replies to authors, not in English, off topic)

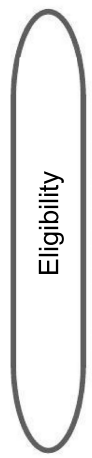

Full-text articles assessed for eligibility

$$
(n=30)
$$

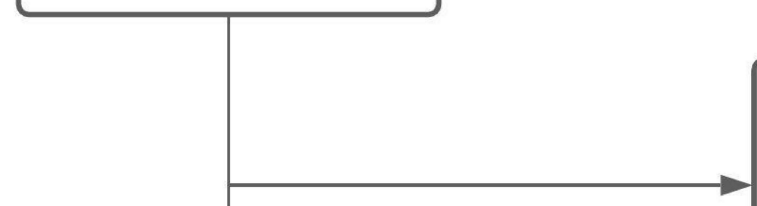

Articles excluded after fulltext evaluation

$$
(n=18)
$$

(irrelevant to research question)

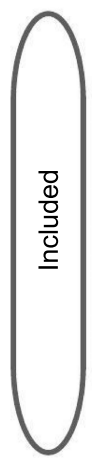

Studies included in review

$(n=12)$

Figure I Summary of the study selection process. 
Table I Summary of Demographics, Baseline Characteristics, and Perioperative Data and the Main Findings of Studies

\begin{tabular}{|c|c|c|c|c|c|c|c|c|c|c|c|c|c|}
\hline $\begin{array}{l}\text { Author and } \\
\text { Year }\end{array}$ & Procedure & $\begin{array}{l}\text { Age } \\
\text { Groups, } \\
\text { Number of } \\
\text { Patients }\end{array}$ & $\begin{array}{l}\text { Age } \\
\text { (Years)* }\end{array}$ & $P V *(m L)$ & $\begin{array}{l}\text { PSA* } \\
\text { (ng/ } \\
\mathrm{mL})\end{array}$ & IPSS* & $\begin{array}{l}\text { Qmax* } \\
(m L / s)\end{array}$ & QoL* & LOS (day)* & OT (min)* & $\begin{array}{l}\text { Post-op } \\
\text { IPSS * }\end{array}$ & $\begin{array}{l}\text { Post-op } \\
\text { Qmax * }\end{array}$ & Main Findings \\
\hline \multirow[t]{2}{*}{$\begin{array}{l}\text { Castellani } \\
\text { et al } 2019^{14} \\
\text { retrospective }\end{array}$} & \multirow[t]{2}{*}{ ThuLEP } & $\geq 75: 129$ & $79.0 \pm 3.7$ & $64(40)$ & $2.9(3)$ & $26(5)$ & $7.8 \pm 2.8$ & $\begin{array}{l}4.5 \\
\pm 0.9\end{array}$ & $3(1)$ & $55(25)$ & $\begin{array}{l}\Delta-19.3 \pm \\
5.0^{\mathrm{b}}(\text { Ist } \\
\text { month })\end{array}$ & N/A & \multirow{2}{*}{$\begin{array}{l}\text { Median operation time, } \\
\text { postoperative hospital stay were } \\
\text { similar in both groups ( } \mathrm{P}=0.452 \text {, } \\
0.547 \text {; respectively). Overall, } \\
85.9 \% \text { of men had no } \\
\text { complications, with no } \\
\text { differences between the groups } \\
(82.5 \% \text { vs } 89.3 \%) \text {. The incidence } \\
\text { of Clavien grade III-IV } \\
\text { complications was comparable } \\
(3.8 \% \text { vs I\%). There were no } \\
\text { statistically significant differences } \\
\text { in IPSS, Qmax, QoL, or } \\
\text { reoperation rate between } \\
\text { groups in I-year follow up. }\end{array}$} \\
\hline & & $<75: 283$ & $65.6 \pm 6.0$ & $55(34)$ & $\begin{array}{l}2.9 \\
(3.2)\end{array}$ & $26(5)$ & $8.1 \pm 2.8$ & $\begin{array}{l}4.3 \\
\pm 0.9\end{array}$ & $3(1)$ & $55(25)$ & $\begin{array}{l}\Delta-18.0 \pm \\
5.6^{\mathrm{b}}(\mathrm{Ist} \\
\text { month) }\end{array}$ & N/A & \\
\hline \multirow{4}{*}{$\begin{array}{l}\text { Piao et al } \\
2016^{6} \\
\text { prospective }\end{array}$} & \multirow[t]{4}{*}{ HoLEP } & 50-59:44 & N/A & $65.6 \pm 50.1$ & $\begin{array}{l}3.5 \pm \\
3.2\end{array}$ & $\begin{array}{l}22.9 \pm \\
6.3\end{array}$ & $8.6 \pm 4.4$ & $\begin{array}{l}4.6 \pm \\
0.8\end{array}$ & $2.3 \pm 0.7$ & $52.3 \pm 30.2$ & $\begin{array}{l}6.2 \pm 6.1 \\
\text { (6th month) }\end{array}$ & $\begin{array}{l}23.6 \pm 13.4 \\
\text { (6th month) }\end{array}$ & \multirow{4}{*}{$\begin{array}{l}\text { Mean operation time, } \\
\text { enucleation time, morcellation } \\
\text { time, and enucleation weight, } \\
\text { hospital stay were higher in } \\
\text { group } \geq 80 \text { compared with other } \\
\text { group patients ( } P=0.002, P= \\
0.010, P<0.01, P=0.009 \text { and } \\
P=0.00 I \text {; respectively). The } \\
\text { incidence of post operative } \\
\text { complications were similar } \\
\text { between groups ( } P>0.05 \text { ). All } \\
\text { the patients in all age groups } \\
\text { showed improvement in } \\
\text { functional outcomes after } \\
\text { HoLEP. No significant difference } \\
\text { was found in terms of IPSS, QoL, } \\
Q \text { max and PVR between groups } \\
\text { at } 6 \text { th month follow uP ( } P> \\
0.05 \text { ). }\end{array}$} \\
\hline & & $60-69: 253$ & $N / A$ & $71.5 \pm 33.8$ & $\begin{array}{l}4.3 \pm \\
4.2\end{array}$ & $\begin{array}{l}19.1 \pm \\
7.3\end{array}$ & $9.0 \pm 4.3$ & $\begin{array}{l}4.2 \pm \\
1.3\end{array}$ & $2.3 \pm 0.7$ & $57.2 \pm 26.5$ & $5.3 \pm 4.8$ & $21.4 \pm 11.4$ & \\
\hline & & $70-79: 244$ & N/A & $68.5 \pm 38.2$ & $\begin{array}{l}3.9 \pm \\
4.2\end{array}$ & $\begin{array}{l}18.3 \pm \\
8.2\end{array}$ & $8.9 \pm 4.8$ & $\begin{array}{l}4.1 \pm \\
1.4\end{array}$ & $2.4 \pm 0.7$ & $59.7 \pm 31.0$ & $6.7 \pm 6.0$ & $19.7 \pm 9.8$ & \\
\hline & & $\geq 80: 38$ & $N / A$ & $86.4 \pm 52.6$ & $\begin{array}{l}4.6 \pm \\
3.5\end{array}$ & $\begin{array}{l}18.5 \pm \\
9.2\end{array}$ & $9.3 \pm 4.5$ & $\begin{array}{l}4.1 \pm \\
1.6\end{array}$ & $2.9 \pm 1.8$ & $79.0 \pm 48.5$ & $5.3 \pm 6.5$ & $17.1 \pm 7.4$ & \\
\hline
\end{tabular}




\begin{tabular}{|c|c|c|c|c|c|c|c|c|c|c|c|c|c|}
\hline \multirow[t]{3}{*}{$\begin{array}{l}\text { Tamalunas } \\
\text { et al } 2020^{21} \\
\text { retrospective }\end{array}$} & \multirow[t]{3}{*}{ HoLEP } & $<70: 208$ & $64(59-67)$ & $\begin{array}{l}75.5(60- \\
100)\end{array}$ & $\begin{array}{l}6(3.2- \\
12.4)\end{array}$ & $\begin{array}{l}20 \\
(15- \\
25)\end{array}$ & $\begin{array}{l}10(7.5- \\
14)\end{array}$ & $\begin{array}{l}4(3- \\
5)\end{array}$ & $3(3.0-3.0)$ & $75(58-109)$ & $\begin{array}{l}\Delta 14(7- \\
17.5)^{\mathrm{b}}(4 \mathrm{th} \\
\text { week) }\end{array}$ & $\begin{array}{l}\Delta 14.5(9- \\
26)^{b}(4 \text { th } \\
\text { week) }\end{array}$ & \multirow{3}{*}{$\begin{array}{l}\text { There was significant } \\
\text { improvement in median IPSS } \\
\text { scores between groups } 4 \text { weeks } \\
\text { after surgery ( } P<0.001) \text {. } \\
\text { A significant improvement was } \\
\text { found in QoL of three points but } \\
\text { no statistically significant } \\
\text { difference between groups (P= } \\
0.63) \text {. Median Qmax showed } \\
\text { significant improvement in all } \\
\text { groups without significant } \\
\text { difference among groups ( } 0.467) \text {. } \\
\text { Perioperative Clavien-Dindo } \\
\text { grade } \geq 11 \text { complications were } \\
\text { found in } 4.1 \% \text { of patients and } \\
\text { there was no significant } \\
\text { difference between groups ( } P= \\
0.176 \text { ). }\end{array}$} \\
\hline & & $70-79: 164$ & $74(72-76)$ & $85(60-120)$ & $\begin{array}{l}6.2(3- \\
11.6)\end{array}$ & $\begin{array}{l}18 \\
(14- \\
23)\end{array}$ & $\begin{array}{l}10(7- \\
13.8)\end{array}$ & $\begin{array}{l}4(3- \\
5)\end{array}$ & $3(3.0-3.0)$ & $\begin{array}{l}87.5(66.8- \\
118)\end{array}$ & $\Delta 10(4-16)^{b}$ & $\begin{array}{l}\Delta 10.5^{\mathrm{b}}(5- \\
16)\end{array}$ & \\
\hline & & $\geq 80: 115$ & $82(80-85)$ & $90(60-115)$ & $\begin{array}{l}5.1 \\
(3.2- \\
11.3)\end{array}$ & $\begin{array}{l}18 \\
(14- \\
25.8)\end{array}$ & $\begin{array}{l}9.5(7.3- \\
12)\end{array}$ & $\begin{array}{l}4(4- \\
5)\end{array}$ & $3(2.5-3.5)$ & $93(57-116)$ & $\begin{array}{l}\Delta 8(0- \\
17.8)^{\mathrm{b}}\end{array}$ & $\begin{array}{l}\Delta 13^{\mathrm{b}}(8.5- \\
17)\end{array}$ & \\
\hline \multirow[t]{4}{*}{$\begin{array}{l}\text { Mmeje et al } \\
2013^{20} \\
\text { retrospective }\end{array}$} & \multirow[t]{4}{*}{ HoLEP } & 50-59:22 & N/A & $29.8 \pm 5.2$ & $\begin{array}{l}3.74 \\
(0.8- \\
15)\end{array}$ & $\begin{array}{l}25.2 \\
\pm 7.6\end{array}$ & $8.1 \pm 3.8$ & N/A & $1.18 \pm 0.5$ & $N / A$ & $\begin{array}{l}7.2 \pm 5.3 \\
\text { (6th month) }\end{array}$ & $\begin{array}{l}19.5 \pm 13.6 \\
\text { (6th month) }\end{array}$ & \multirow{4}{*}{$\begin{array}{l}\text { No significant difference was } \\
\text { found in terms of IPSS, Qmax, or } \\
\text { PVR between groups at I year } \\
\text { follow up ( } \mathrm{p}=0.364, \mathrm{p}=0.082 \text {, } \\
\mathrm{p}=0.69) \text {. Mean hospital stay was } \\
\text { similar between groups } \\
(\mathrm{p}=0 . \mathrm{I} \text { I } 2) \text {. There was no } \\
\text { significant difference regarding } \\
30 \text {-day complications in Clavien- } \\
\text { Dindo system ( } \mathrm{p}=0.9) \text {. Clavien } \\
\text { grade } \geq \mathrm{III} \text { complications was } \\
\text { minimal in all groups }(0 \%, 5.6 \% \text {, } \\
3.9 \% \text { and } 4.4 \% \text { in groups). }\end{array}$} \\
\hline & & $60-69: 91$ & $N / A$ & $28.9 \pm 4.7$ & $\begin{array}{l}3.20 \\
(0.28- \\
21.74)\end{array}$ & $\begin{array}{l}21.2 \\
\pm 7.7\end{array}$ & $9.4 \pm 6.1$ & N/A & $1.28 \pm 0.7$ & N/A & $5 \pm 4.4$ & $24.7 \pm 11$ & \\
\hline & & 70-79:153 & $N / A$ & $27.2 \pm 3.9$ & $\begin{array}{l}4.63 \\
(0.37- \\
91.2)\end{array}$ & $\begin{array}{l}19.5 \\
\pm 6.6\end{array}$ & $9.7 \pm 6.3$ & N/A & $1.26 \pm 0.9$ & $N / A$ & $5.7 \pm 4.7$ & $20.3 \pm 8.9$ & \\
\hline & & $\geq 80: 45$ & N/A & $25.8 \pm 3.2$ & $\begin{array}{l}6.20 \\
(0.67- \\
82.7)\end{array}$ & $\begin{array}{l}23.3 \\
\pm 7.3\end{array}$ & $9.1 \pm 5.0$ & N/A & $1.68 \pm 2.1$ & N/A & $8.7 \pm 8.3$ & $15.1 \pm 6.7$ & \\
\hline
\end{tabular}

(Continued) 
Table I (Continued).

\begin{tabular}{|c|c|c|c|c|c|c|c|c|c|c|c|c|c|}
\hline $\begin{array}{l}\text { Author and } \\
\text { Year }\end{array}$ & Procedure & $\begin{array}{l}\text { Age } \\
\text { Groups, } \\
\text { Number of } \\
\text { Patients }\end{array}$ & $\begin{array}{l}\text { Age } \\
\text { (Years)* }\end{array}$ & $\mathbf{P V}^{*}(\mathrm{~mL})$ & $\begin{array}{l}\text { PSA* } \\
(\mathbf{n g} / \\
\mathrm{mL})\end{array}$ & IPSS* & $\begin{array}{l}\text { Qmax* } \\
(\mathrm{mL} / \mathrm{s})\end{array}$ & QoL* & LOS (day)* & OT $(\mathrm{min})^{*}$ & $\begin{array}{l}\text { Post-op } \\
\text { IPSS * }\end{array}$ & $\begin{array}{l}\text { Post-op } \\
\text { Qmax * }\end{array}$ & Main Findings \\
\hline \multirow{4}{*}{$\begin{array}{l}\text { Gild et al } \\
2020^{17} \\
\text { retrospective }\end{array}$} & \multirow[t]{4}{*}{ HoLEP } & $\leq 60: 134$ & \multirow[t]{4}{*}{$71(66-76)$} & \multirow[t]{4}{*}{$80(58-105)$} & \multirow{4}{*}{$\begin{array}{l}5.3 \\
(2.8- \\
10)\end{array}$} & \multirow{4}{*}{$\begin{array}{l}19 \\
(13- \\
24)\end{array}$} & \multirow{4}{*}{$\begin{array}{l}10(7- \\
14)\end{array}$} & \multirow{4}{*}{$\begin{array}{l}4(3- \\
5)\end{array}$} & \multirow[t]{4}{*}{ N/A } & \multirow[t]{4}{*}{ N/A } & \multirow[t]{4}{*}{ N/A } & $\Delta I 8(9-27)^{b}$ & \multirow{4}{*}{$\begin{array}{l}\text { The improvement in maximal } \\
\text { flow rate was more pronounced } \\
\text { in men under } 60 \text { years than in } \\
\text { men over } 80 \text { [I2 (IQR: } 3.5-18 \text { ) } \\
\text { vs. } 18 \mathrm{~mL} / \mathrm{s}(\mathrm{IQR}: 9.0-27)] \text {. The } \\
\text { improvement in PVR was more } \\
\text { pronounced in men aged } 80 \\
\text { years and older than men<60 } \\
\text { years [(100 } \mathrm{mL} \text { (IQR: } 34-233 \text { ) vs } \\
85 \mathrm{~mL} \text { (IQR: } 35-180)] \text {. There } \\
\text { were significant differences in } \\
\text { terms of prolonged } \\
\text { catheterization and perioperative } \\
\text { blood transfusion ( } \mathrm{p}=0.04, \\
\mathrm{p}<0.001 \text {; respectively). } \\
\text { Postoperative complications } \\
\text { (ClavienDindo grade } \geq 3 \mathrm{~b} \text { were } \\
\text { seen as } 7.1 \% \text { in patients with } \geq \\
80 \text { years and there was } \\
\text { a significant difference between } \\
\text { groups ( }=0.02) \text { ). }\end{array}$} \\
\hline & & $60-69: 636$ & & & & & & & & & & $\begin{array}{l}\Delta 13(6.2- \\
21)^{\mathrm{b}}\end{array}$ & \\
\hline & & $70-79: 862$ & & & & & & & & & & $\begin{array}{l}\Delta 10(3.7- \\
19)^{\mathrm{b}}\end{array}$ & \\
\hline & & $\geq 80: 184$ & & & & & & & & & & $\begin{array}{l}\Delta 12(3.5- \\
18)^{\mathrm{b}}\end{array}$ & \\
\hline \multirow{4}{*}{$\begin{array}{l}\text { Elshal et al } \\
2013^{16} \\
\text { retrospective }\end{array}$} & HoLEP & \multirow[t]{4}{*}{$\geq 80: 264$} & $83.9 \pm 3.3$ & $94.4 \pm 59$ & $\begin{array}{l}15.1 \pm \\
28.2\end{array}$ & \multirow[t]{4}{*}{ N/A } & \multirow[t]{4}{*}{ N/A } & \multirow[t]{4}{*}{$N / A$} & \multirow[t]{4}{*}{$1.2 \pm 1.2$} & $91.9 \pm 42$ & $\begin{array}{l}4.7 \text { (12th } \\
\text { month) }\end{array}$ & $\begin{array}{l}18 \text { (12th } \\
\text { month) }\end{array}$ & \multirow{4}{*}{ 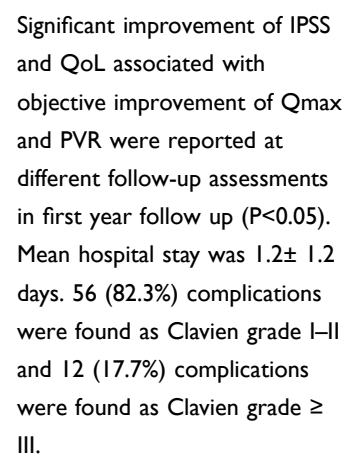 } \\
\hline & HoLAP & & $84.19 \pm 3.7$ & $32.9 \pm 15$ & $\begin{array}{l}13.1 \pm \\
37\end{array}$ & & & & & $54 \pm 33$ & 10.2 & 12.9 & \\
\hline & Hol-TUIP & & $84.3 \pm 3.6$ & $32.4 \pm 12$ & $\begin{array}{l}4.8 \pm \\
7.9\end{array}$ & & & & & $31.3 \pm 18$ & 6.5 & 19.8 & \\
\hline & PVP & & $84.2 \pm 3.8$ & $38.3 \pm 24$ & $\begin{array}{l}14.6 \pm \\
42\end{array}$ & & & & & $67.4 \pm 45$ & 8.8 & 16 & \\
\hline
\end{tabular}




\begin{tabular}{|c|c|c|c|c|c|c|c|c|c|c|c|c|c|}
\hline $\begin{array}{l}\text { Liu et al } \\
2020^{19} \\
\text { retrospective }\end{array}$ & $\begin{array}{l}\text { GreenLight } \\
\text { XPS I80-W } \\
\text { PVP }\end{array}$ & $>80: 150$ & $84.7 \pm 2.8$ & $65 \pm 12.5$ & $\begin{array}{l}3.0 \\
\pm 2.1\end{array}$ & $\begin{array}{l}22.5 \\
\pm 4.1\end{array}$ & $7.5 \pm 2.4$ & $\begin{array}{l}4.3 \\
\pm 0.7\end{array}$ & $3.5 \pm 2.1$ & $63 \pm 12.5$ & $\begin{array}{l}10.8 \pm 2.9 \\
\text { (12th } \\
\text { month) }\end{array}$ & $\begin{array}{l}8.7 \pm 5.1 \\
(12 \text { th } \\
\text { month) }\end{array}$ & $\begin{array}{l}\text { There were significant } \\
\text { improvements in IPSS, QOL, } \\
\text { Qmax, and PVR. Intraoperative, } \\
\text { short term and long term } \\
\text { postoperative complication rates } \\
\text { were found as } 0.7 \%, 29.8 \% \text {, and } \\
13.3 \% \text {, respectively. }\end{array}$ \\
\hline $\begin{array}{l}\text { Hou et al } \\
2016^{18} \\
\text { retrospective }\end{array}$ & ThuVEP & $>80: 38$ & $82.8 \pm 2.4$ & $67.2 \pm 27.8$ & $\begin{array}{l}8.4 \\
\pm 6.1\end{array}$ & $\begin{array}{l}24.9 \\
\pm 5.0\end{array}$ & $8.6 \pm 4.3$ & N/A & $N / A$ & $84.7 \pm 32.3$ & $N / A$ & 2.3 & $\begin{array}{l}\text { In the elderly group (age }>80) \text {, no } \\
\text { significant difference was found } \\
\text { regarding Qmax increase from } \\
\text { baseline ( } \mathrm{p}=0.103) \text {. In terms of } \\
\text { PVR decrease, there was no } \\
\text { significant difference from } \\
\text { baseline ( } \mathrm{p}=0.068) \text {. No prostate } \\
\text { capsule perforation and bladder } \\
\text { wall injury during morcellation } \\
\text { was seen. No blood transfusion } \\
\text { or recatheterization were } \\
\text { required in any patient. }\end{array}$ \\
\hline
\end{tabular}

(Continued) 
Table I (Continued).

\begin{tabular}{|c|c|c|c|c|c|c|c|c|c|c|c|c|c|}
\hline $\begin{array}{l}\text { Author and } \\
\text { Year }\end{array}$ & Procedure & $\begin{array}{l}\text { Age } \\
\text { Groups, } \\
\text { Number of } \\
\text { Patients }\end{array}$ & $\begin{array}{l}\text { Age } \\
\text { (Years)* }\end{array}$ & $P V *(m L)$ & $\begin{array}{l}\text { PSA* } \\
(\mathrm{ng} / \\
\mathrm{mL})\end{array}$ & IPSS* & $\begin{array}{l}\text { Qmax* } \\
(m L / s)\end{array}$ & QoL* & LOS (day)* & OT (min)* & $\begin{array}{l}\text { Post-op } \\
\text { IPSS * }\end{array}$ & $\begin{array}{l}\text { Post-op } \\
\text { Qmax * }\end{array}$ & Main Findings \\
\hline \multirow{2}{*}{$\begin{array}{l}\text { Ánan et al } \\
2021^{22} \\
\text { prospective }\end{array}$} & \multirow[t]{2}{*}{ HoLEP } & $\geq 75: 38$ & $79.3 \pm 3.0$ & $70 \pm 37$ & $\begin{array}{l}6.0 \\
\pm 5.6\end{array}$ & $\begin{array}{l}19.6 \\
\pm 7.9\end{array}$ & $8.1 \pm 4.8$ & $\begin{array}{l}6.3 \\
\pm 0.7\end{array}$ & $4 \pm 0.3$ & $97 \pm 31$ & $\begin{array}{l}5.1 \pm 3.3 \text { (6th } \\
\text { month) }\end{array}$ & $\begin{array}{l}17.0 \pm 9.2 \\
\text { (6th month) }\end{array}$ & \multirow[b]{2}{*}{ 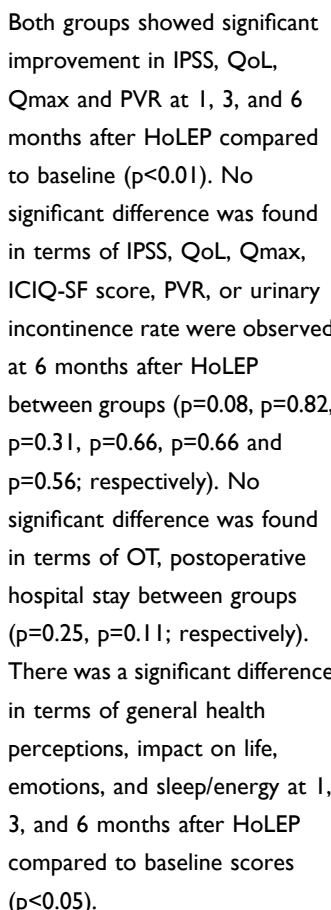 } \\
\hline & & $<75: 62$ & $69.2 \pm 4.2$ & $65 \pm 27$ & $\begin{array}{l}5.9 \\
\pm 4.6\end{array}$ & $\begin{array}{r}18.9 \\
\pm 8.6\end{array}$ & $8.7 \pm 3.4$ & $\begin{array}{l}6.1 \\
\pm 1.0\end{array}$ & $4 \pm 0.5$ & $90 \pm 30$ & $3.9 \pm 2.6$ & $19.3 \pm 9.8$ & \\
\hline
\end{tabular}




\begin{tabular}{|c|c|c|c|c|c|c|c|c|c|c|c|c|c|}
\hline \multirow[t]{2}{*}{$\begin{array}{l}\text { Majumdar } \\
\text { et al } 2014^{24} \\
\text { prospective }\end{array}$} & \multirow[t]{2}{*}{$\begin{array}{l}\text { KTP } \\
80-180 \\
\text { W PVP }\end{array}$} & $<75: 153$ & N/A & N/A & $\begin{array}{l}2.3 \\
(1.4- \\
4)\end{array}$ & $\begin{array}{l}19 \\
(14- \\
24)\end{array}$ & N/A & 4 & 0 & N/A & $\begin{array}{l}\Delta 5.5^{\mathrm{b}} \text { (Ist } \\
\text { Month) }\end{array}$ & N/A & \multirow{2}{*}{$\begin{array}{l}\text { In the Ist month follow-up, there } \\
\text { was a significant improvement in } \\
\text { median IPSS in both age groups } \\
(23 \text { to } 10 \text { years in the young } \\
\text { group and from } 19 \text { to } 16 \text { in the } \\
\text { elderly group; } P<0.001 \text { and } P= \\
0.01 \text {, respectively), while the IPSS } \\
\text { score in the elderly group was } \\
\text { found to be significantly higher } \\
\text { than the younger group ( } 16 \text { vs } \\
10 ; P=0.01) \text {. They found no } \\
\text { significant difference between } \\
\text { age groups in terms of } \\
\text { perioperative complications. }\end{array}$} \\
\hline & & $\geq 75: 49$ & N/A & N/A & $\begin{array}{l}3(1.4- \\
5.7)\end{array}$ & $\begin{array}{l}23 \\
(17- \\
27)\end{array}$ & N/A & 4 & 1 & N/A & $\Delta 9^{\mathrm{b}}$ & N/A & \\
\hline \multirow{2}{*}{$\begin{array}{l}\text { Gu et al } \\
2012^{23} \\
\text { prospective }\end{array}$} & \multirow[t]{2}{*}{$\begin{array}{l}\text { GreenLight } \\
\text { HPS TM PVP }\end{array}$} & $<70: 93$ & $61.0 \pm 6.6$ & $58.7 \pm 32$ & $\begin{array}{l}1.9 \pm \\
2.0\end{array}$ & $\begin{array}{l}23.2 \pm \\
6.3^{2}\end{array}$ & $9.4 \pm 3.9$ & $\begin{array}{l}4.6 \pm \\
1.1\end{array}$ & N/A & $30.9 \pm 25.3$ & $3.1 \pm 1.6^{\mathrm{a}}$ & $20.5 \pm 3.8$ & \multirow[b]{2}{*}{$\begin{array}{l}\text { Significant improvements were } \\
\text { observed in American Urological } \\
\text { Association Symptom Score } \\
\text { (AUASS), QoL, Qmax and PVR } \\
\text { compared to baseline values in } \\
\text { the 3rd, 6th, I } 2 \text { th, and } 36 \text { th } \\
\text { month controls in both age } \\
\text { groups (p< } 0.05 \text { ). There was no } \\
\text { significant difference between } \\
\text { age groups in terms of these } \\
\text { parameters. } \\
\text { There was no significant } \\
\text { difference between age groups } \\
\text { regarding perioperative } \\
\text { complications. No postoperative } \\
\text { urinary incontinence was } \\
\text { observed in either age group. } \\
\text { Bladder neck contraction and } \\
\text { urethral stricture, which are late } \\
\text { complications after the } \\
\text { postoperative Ist month, were } \\
\text { not observed in either group. }\end{array}$} \\
\hline & & $\geq 70: 71$ & $75.9 \pm 4$ & $73.6 \pm 39$ & $\begin{array}{l}2.9 \pm \\
2.4\end{array}$ & $\begin{array}{l}21.8 \pm \\
6.4^{a}\end{array}$ & $9.1 \pm 3.6$ & $\begin{array}{l}4.5 \pm \\
1.0\end{array}$ & N/A & $36.1 \pm 21.7$ & $3.2 \pm 1.6^{\mathrm{a}}$ & $18.9 \pm 3.9$ & \\
\hline
\end{tabular}

(Continued) 
Table I (Continued).

\begin{tabular}{|c|c|c|c|c|c|c|c|c|c|c|c|c|c|}
\hline $\begin{array}{l}\text { Author and } \\
\text { Year }\end{array}$ & Procedure & $\begin{array}{l}\text { Age } \\
\text { Groups, } \\
\text { Number of } \\
\text { Patients }\end{array}$ & $\begin{array}{l}\text { Age } \\
\text { (Years)* }\end{array}$ & PV* (mL) & $\begin{array}{l}\text { PSA* } \\
\text { (ng/ } \\
\mathrm{mL})\end{array}$ & IPSS* & $\begin{array}{l}\operatorname{Qmax}^{*} \\
(\mathrm{~mL} / \mathrm{s})\end{array}$ & QoL* & LOS (day)* & OT $(\mathrm{min}) *$ & $\begin{array}{l}\text { Post-op } \\
\text { IPSS * }\end{array}$ & $\begin{array}{l}\text { Post-op } \\
\text { Qmax * }\end{array}$ & Main Findings \\
\hline \multirow{2}{*}{$\begin{array}{l}\text { Bouhadana } \\
\text { et al } 2021^{15} \\
\text { retrospective }\end{array}$} & TURP & $\geq 75: 12,262$ & $80.7 \pm 4.5$ & \multirow[t]{2}{*}{$N / A$} & \multirow[t]{2}{*}{ N/A } & \multirow[t]{2}{*}{$N / A$} & \multirow[t]{2}{*}{$N / A$} & \multirow[t]{2}{*}{$N / A$} & \multirow[t]{2}{*}{ N/A } & \multirow[t]{2}{*}{ N/A } & \multirow[t]{2}{*}{$\mathrm{N} / \mathrm{A}$} & \multirow[t]{2}{*}{ N/A } & \multirow[b]{2}{*}{$\begin{array}{l}\text { TURP was found to be } \\
\text { associated with higher rates of } \\
90 \text {-day hospital readmission and } \\
\text { probability of emergency room } \\
\text { visits (OR } 1.07 \text {, \%95 Cl). In } \\
\text { addition, post-TURP hematuria } \\
\text { is more likely to occur (OR I.28 } \\
\% 95 \mathrm{Cl} 1.03-1.59 \text { ). However, } \\
\text { TURP has also been found to } \\
\text { reduce the risk of reoperation by } \\
20 \% \text { in } 6 \text { months or longer (\%95 } \\
\text { Cl } 0.71-0.91 \text { ). }\end{array}$} \\
\hline & $\begin{array}{l}\text { Laser } \\
\text { prostatectomy }\end{array}$ & 275: 17,544 & $80.8 \pm 4.6$ & & & & & & & & & & \\
\hline
\end{tabular}

Note: *Mean \pm SD or median (range) (IQR).

Abbreviations: HoLEP, Holmium laser enucleation of the prostate; ThuLEP, Thulium laser enucleation of the prostate; HoLAP, Holmium laser ablation of the prostate; Hol-TUIP, Holmium laser transurethral incision of the prostate; PVP, Photoselective vaporization of the prostate; ThuVEP, Thulium vapoenucleation of the prostate; KTP, potassium-titanyl-phosphate; TURP, Transurethral resection of the prostate; PV, prostate volume; PSA, prostate specific antigen; IPSS, International Prostate Symptom Score; ICIQ-SF, International Consultation on Incontinence Questionnaire-Short Form; Qmax, maximum urinary flow rate; QoL, quality of life; LOS, Length of hospital stay; OT, operation time; SD, standard deviation; $\mathrm{Cl}$, confidence interval; OR, odds ratio; N/A, not applicable. ${ }^{a} \mathrm{AUASS}$, American Urological Association Symptom Score; ${ }^{\mathrm{b}} \Delta$, Change from baseline. 


\section{Perioperative and Urinary Outcomes} Studies Comparing Men Aged $\geq 75$ Years vs $<75$ Years

Castellani et al compared the outcomes of ThuLEP in men aged $\geq 75$ years vs $<75$ years in a retrospective study including 412 men. ${ }^{14}$ They observed no statistically significant difference in median operation times and LOS ( $p=0.452, p=0.547$; respectively) between groups. There were no differences in IPSS, Qmax, QoL, or reoperation rate between groups at the 1-year follow up. ${ }^{14}$

Majumdar et al investigated prospectively the efficacy and safety of laser photoselective vaporization (LVP) in groups aged $\geq 75(n=49)$ and $<75$ years $(n=153) .{ }^{24}$ In contrast to Castellani et al, the $\geq 75$ age group's median LOS was significantly longer ( 1 day vs 0 days; $\mathrm{P}=0.001$ ). In the 1 st month follow-up, there was a significant improvement in median IPSS in both age groups (23 to 10 years in the younger group and from 19 to 16 in the elderly group; $\mathrm{P}<$ 0.001 and $\mathrm{P}=0.01$, respectively), while the elderly group's IPSS was significantly higher than the younger group's (16 vs $10 ; \mathrm{P}=0.01)$. However, no significant difference was observed between the groups in median IPSS at the postoperative 3rd-, 6th- and 9th-month follow-ups.

Anan et al prospectively compared perioperative outcomes, complications and the King's Health Questionnaire (KHQ scores) of 100 patients aged $\geq 75$ years and $<75$ years after HoLEP. ${ }^{22}$ KHQ is a special internationally validated QoL questionnaire containing the following 9 categories: health perceptions, physical limitations, role limitations, impact on life, social limitations, personal relationships, emotions, sleep/ energy, and incontinence status. Scores in each category range from 0 to 100, with lower scores indicating better QoL. No significant differences were detected in IPSS, QoL, Qmax, International Consultation on Incontinence QuestionnaireShort Form (ICIQ-SF-Score), operative times, LOS, and urinary incontinence rates between the groups 6 months after HoLEP. Regarding the KHQ categories, general health perceptions, emotions, impact of life and sleep/energy 1, 3 and 6 months after HoLEP; role limitations, physical limitations, and social limitations 3 and 6 months after HoLEP; and personal relationships and incontinence severity 6 months after HoLEP were better in both age groups than at baseline levels $(\mathrm{P}<0.05) .{ }^{22}$ There were no significant differences between groups.

Bouhadana et al compared the safety and durability of TURP $(n=12262)$ and laser prostatectomy (LP) $(n=17544)$ in men aged $\geq 75$ and multi-morbid patients. ${ }^{15}$ However, their study provides no results on the functional outcomes of surgical procedures.

\section{Studies Comparing Different Age Groups}

Piao et al compared outcomes in groups aged 50-59 $(\mathrm{n}=44), 60-69 \quad(\mathrm{n}=253), 70-79 \quad(\mathrm{n}=244)$, and $\geq 80$ years $(n=38)$. Patients aged $\geq 80$ years revealed the longest LOS (group A, $2.3 \pm 0.7$ days; group $\mathrm{B}, 2.3 \pm 0.7$ days vs group C, $2.4 \pm 0.7$ days; group $\mathrm{D}, 2.9 \pm 1.8$ days, $\mathrm{p}=0.001){ }^{6}$ Functional outcomes were significantly better than conditions at baseline, and there were no differences in IPSS, QoL, Qmax, and postvoid residual volume (PVR) among these groups at the 6-month follow-up $(p>0.05){ }^{6}$ Tamalunas et al retrospectively analysed 487 patients who underwent HoLEP. ${ }^{21}$ Their study cohort was divided into 3 groups according to age $(<70$ years, $70-79$ years and $\geq 80$ years). At the 1 -month follow-up, all groups revealed significantly higher median IPSS scores $(p<0.001)$. Significant improvement was identified in QoL and Qmax, but without any differences between age groups ( $\mathrm{p}=0.63$ and $\mathrm{p}=0.467$, respectively). ${ }^{21}$ Mmeje et al assessed outcomes after HoLEP in 311 patients according to age groups (22 patients aged 50-59 years, 91 aged 60 69 years, 153 aged $70-79$ years, and $45 \geq 80$ years). ${ }^{20}$ They reported no significant difference in terms of IPSS, Qmax, PVR and LOS between groups at their 1-year follow-up. ${ }^{20}$

$\mathrm{Gu}$ et al prospectively evaluated the efficacy and safety of GreenLight HPS ${ }^{\mathrm{TM}}$ (High Performance System) laser photoselective vaporisation prostatectomy (PVP) in male groups aged $<70(n=93)$ and $\geq 70(n=71) .{ }^{23}$ They observed significant improvements in the American Urological Association Symptom Score (AUASS), QoL, Qmax and PVR compared to baseline values at the 3rd-, 6th-, 12th-, and 36th-month follow-ups in both age groups $(p<0.05)$. They reported no significant difference between age groups in those parameters.

\section{Studies Including Men Aged $\geq 80$ Years}

Gild et al performed a retrospective analysis including of 1816 men's perioperative outcomes after HoLEP. ${ }^{17}$ Their study emphasises that the Qmax improvement was more pronounced in men aged under 60 years than in those over 80 [12 (IQR: $3.5-18)$ vs $18 \mathrm{~mL} / \mathrm{s}$ (IQR: 9.0-27)], whereas the improvement in PVR was more pronounced in men aged 80 years and older than those $<60$ years $[100 \mathrm{~mL}$ (IQR: 34-233) vs $85 \mathrm{~mL}$ (IQR: 35-180)]. Prolonged catheterisation was not associated with patient age. ${ }^{17}$ Elshal et al compared the outcomes of 264 octogenarians 
(>80 years) who underwent various prostate laser surgeries [HoLEP, HoLAP (holmium laser ablation of the prostate), Hol-TUIP (holmium laser transurethral incision of the prostate) and PVP (photoselective vaporization]. ${ }^{16}$ Significant improvements in IPSS and QoL scores and improvements in Qmax and PVR were reported in all groups in the first year. ${ }^{16}$ Liu et al retrospectively investigated the safety and effectiveness of GreenLight XPS 180$\mathrm{W}$ laser photoselective vaporisation of the prostate (PVP) in 150 elderly patients (>80 years). ${ }^{19}$ IPSS, QOL, Qmax, and PVR scores rose significantly after surgery at months 3,6 , and 12 when compared to baseline. ${ }^{19}$ Hou et al retrospectively analysed the results of 303 patients who underwent thulium vapoenucleation of the prostate (ThuVEP). ${ }^{18}$ The older group's (age>80) Qmax did not differ significantly from the younger groups' $(p=0.103) .^{18}$

\section{Comorbidities}

Table 2 illustrates ASA scores, AP/AC treatment status and comorbidities sorted by age groups of patients included in the studies.

Castellani et al reported that patients $\geq 75$ years underwent AP/AC therapy more frequently than $<75$-year-old patients $(20.2 \%$ vs $7.1 \% ; p<0.05)$. Age groups $\geq 75$ years had ASA scores 3-4 more frequently than age groups $<75$ $(57.4 \%$ vs $17.0 \% ; \mathrm{p}<0.05) .{ }^{14}$ In the study by Majumdar et al, heart disease, haematuria and use of anticoagulants were more common in $\geq 75$ age group than their younger group $(p=0.03, p=0.05, p=0.002) .^{24}$ Anan et al reported that comorbidities were more likely in groups aged $\geq 75$ years. $^{22}$ There was no significant difference between groups in $\mathrm{AC}$ therapy, $(21 \%$ vs $34 \% ; \mathrm{p}=0.16)$. There was no significant difference in ASA scores in the study by $\mathrm{Gu}$ et al, $(2.3 \pm 0.6$ vs $2.4 \pm 0.7 ; \mathrm{p}=0.326)$ or in anticoagulation use $(36.6 \%$ vs $26.8 \% ; \mathrm{p}=0.238)$ in $<70$ and $\geq 70$ age groups. $^{23}$

On the other hand, Tamalunas et al and Mmeje et al demonstrated that $\geq 80$-year-old patients were more likely to undergo $\mathrm{AP} / \mathrm{AC}$ therapy than younger age groups (39.1\% and $53.3 \%$, respectively). ${ }^{20,21}$ They found that ASA $\geq 3$ was higher in $\geq 80$-year-old patients than in younger patients $(\geq 80: 65.2 \%$; <70: 24.9\%; 70-79: $28.7 \%)^{21}$ Mmeje et al reported that $>80$-year-olds had the highest ASA scores among age groups (50-59: $\mathrm{n}=2.18 ; 60-69: \mathrm{n}=2.3 ; 70-79: \mathrm{n}=2.46 ;>80: \mathrm{n}=2.6) .^{20}$ Piao et al also showed that patients $\geq 80$ years had significantly higher ASA scores $(\mathrm{p}=0.006)$ and higher anticoagulation use $(p=0.008)$ prior to surgery. ${ }^{6}$ Liu et al reported that most patients over 80 years old received an ASA score of 2 or $3(45.4 \%$ and $44 \%)$. The patients included in their study probably had cardiovascular disease $(61.4 \%)$, followed by hypertension $(43.4 \%){ }^{19}$

\section{Complications}

Table 3 illustrates perioperative complications and adverse events according to Clavien- Dindo classification of the patients included in the study stratified by age.

Castellani et al identified comparable incidences of complications according to Clavien- Dindo classification between age groups ( $\geq 75$ vs $<75$ years) $(p=0.743)$. The vast majority had no complications $(85.9 \%)$, with no differences between age groups (82.5\% vs $89.3 \%$ ) for complications such as urethral stenosis and bladder-neck contracture. ${ }^{14}$ They also found that urinary incontinence (UI) rates were similar in patients $\geq 75$ and $<75$ years in their 1-year follow-up (5.8\% vs 4.8\%, respectively).

The study of Majumdar et al reported that the most common perioperative complications in the $\geq 75$ age group were dysuria $(10.2 \%)$, urinary tract infection $(10.2 \%)$, and haematuria $(10.2 \%){ }^{24}$ They detected no significant difference between age groups in perioperative complications.

Anan et al reported postoperative fever at similar rates in their groups $\geq 75$ years and $<75$ years ( $3 \%$ vs $2 \%$; $p=1.00) .^{22}$ They observed a higher rate of UI in the $\geq 75$ years group at the postoperative 6th-month follow-up than in the younger group, but the difference was not significant ( $5 \%$ vs $2 \%, \mathrm{p}=0.56)$.

Bouhadana et al found that TURP was associated with higher rates of 90-day hospital readmission and probability of emergency room visits than laser prostatectomy in their patients aged $\geq 75$ (OR 1.07, \%95 CI). ${ }^{15}$ Post-TURP haematuria was also more likely (OR 1.28, \%95 CI 1.031.59). However, TURP was also found to reduce the risk of reoperation by $20 \%$ at 6 months postoperatively or later (\%95 CI 0.71-0.91). ${ }^{15}$

$\mathrm{Gu}$ et al reported that the most common 1st-month postoperative complications were haematuria (lasting under 7 days) (64.8\%), de novo retrograde ejaculation $(21.2 \%)$, urinary tract infection $(4.2 \%)$, and transient irritative voiding symptoms $(4.2 \%)$ in the $\geq 70$ years group after Greenlight PVP. ${ }^{23}$ They detected no significant difference between age groups in perioperative complications, nor was any postoperative UI observed in either age group. Bladder-neck contracture and urethral stricture, which are late complications after the postoperative 1st month, were not observed in either group. 
Table 2 ASA Scores, AP/AC Treatment Status and Comorbidities According to Age Groups of the Patients Included in the Studies

\begin{tabular}{|c|c|c|c|c|c|c|c|}
\hline \multirow{2}{*}{$\begin{array}{l}\text { Author and } \\
\text { Year }\end{array}$} & \multirow{2}{*}{$\begin{array}{l}\text { Age Groups } \\
\text { (Years) }\end{array}$} & \multirow{2}{*}{$\begin{array}{l}\text { ASA Score, } \\
n(\%)\end{array}$} & \multirow{2}{*}{$\begin{array}{l}\text { APIAC } \\
\text { Therapy, n(\%) }\end{array}$} & \multicolumn{4}{|c|}{ Comorbidities } \\
\hline & & & & DM, n(\%) & HT, n(\%) & ND, $n(\%)$ & CVD, n(\%) \\
\hline \multirow{6}{*}{$\begin{array}{l}\text { Castellani et al } \\
2019^{14}\end{array}$} & \multirow[t]{3}{*}{$\geq 75$} & I: I4 (I0.9) & \multirow[t]{3}{*}{$26(20.2)$} & \multirow{3}{*}{\multicolumn{4}{|c|}{ N/A }} \\
\hline & & 2: 41 (3I.8) & & & & & \\
\hline & & 3-4: 74 (57.4) & & & & & \\
\hline & \multirow[t]{3}{*}{$<75$} & I: IIO (38.9) & \multirow[t]{3}{*}{$20(7.1)$} & \multirow{3}{*}{\multicolumn{4}{|c|}{$\mathrm{N} / \mathrm{A}$}} \\
\hline & & 2: $125(44.2)$ & & & & & \\
\hline & & 3-4: $48(17.0)$ & & & & & \\
\hline \multirow[t]{4}{*}{ Piao et al $2016^{6}$} & $50-59$ & $1.5 \pm 0.5^{*}$ & $6(13.6)$ & $5(11.4)$ & II (25.0) & I (2.3) & $3(6.8)$ \\
\hline & $60-69$ & $1.7 \pm 0.5 *$ & $56(22.1)$ & $46(18.2)$ & $104(41.3)$ & $30(11.9)$ & $20(7.9)$ \\
\hline & $70-79$ & $1.7 \pm 0.5^{*}$ & $79(32.4)$ & $49(20.1)$ & II 8 (48.4) & 31 (12.7) & $17(7.0)$ \\
\hline & $\geq 80$ & $1.8 \pm 0.5^{*}$ & $13(34.2)$ & $8(2 I . I)$ & $22(57.9)$ & $6(15.8)$ & $4(10.5)$ \\
\hline \multirow{3}{*}{$\begin{array}{l}\text { Tamalunas et al } \\
2020^{21}\end{array}$} & $<70$ & 23: 5 I (24.9) & $30(14.4)$ & \multirow{11}{*}{\multicolumn{4}{|c|}{$\mathrm{N} / \mathrm{A}$}} \\
\hline & $70-79$ & $\geq 3: 46$ (28.7) & $33(20.1)$ & & & & \\
\hline & $\geq 80$ & 23: 75 (65.2) & $45(39.1)$ & & & & \\
\hline \multirow{4}{*}{$\begin{array}{l}\text { Mmeje et al } \\
2013^{20}\end{array}$} & $50-59$ & $2.18 \pm 0.50^{*}$ & $2(9.1)$ & & & & \\
\hline & $60-69$ & $2.30 \pm 0.55^{*}$ & $33(36.3)$ & & & & \\
\hline & $70-79$ & $2.46 \pm 0.55^{*}$ & $68(43.4)$ & & & & \\
\hline & $\geq 80$ & $2.6 \pm 0.54^{*}$ & $24(53.3)$ & & & & \\
\hline \multirow{4}{*}{$\begin{array}{l}\text { Gild et al } \\
2020^{17}\end{array}$} & $\leq 60$ & \multirow{4}{*}{$\begin{array}{l}\text { I: I36 (7.5) } \\
\text { 2: } 1007(56) \\
\text { 3: } 60 \text { I (33) } \\
\text { 4: I7 (0.9) } \\
\text { Unknown: } 55 \\
(3.0)\end{array}$} & \multirow[t]{4}{*}{$803(44.2)$} & & & & \\
\hline & $60-69$ & & & & & & \\
\hline & $70-79$ & & & & & & \\
\hline & $\geq 80$ & & & & & & \\
\hline $\begin{array}{l}\text { Elshal et al } \\
2013^{16}\end{array}$ & $\geq 80$ & $\begin{array}{l}\geq 3: \text { I04 }(60.8) \text { for } \\
\text { HoLEP } \\
\geq 3: 10(62.5) \text { for } \\
\text { HoLAP } \\
\geq 3: 8(61.5) \text { for } \\
\text { Hol-TUIP } \\
\geq 3: 42(65.6) \text { for } \\
\text { PVP }\end{array}$ & $\begin{array}{l}66(38.5) \text { for } \\
\text { HoLEP } \\
3 \text { (I8.7) for } \\
\text { HoLAP } \\
3 \text { (23) for Hol- } \\
\text { TUIP } \\
\text { I9 (29.6) for } \\
\text { PVP }\end{array}$ & $\begin{array}{l}\text { I2 (7) for HoLEP } \\
\text { I (6.5) for HoLAP } \\
7 \text { (53.8) for Hol-TUIP } \\
2 \text { (3.1) for PVP }\end{array}$ & $\mathrm{N} / \mathrm{A}$ & $N / A$ & $\begin{array}{l}2 \text { I }(12.2) \text { for } \\
\text { HoLEP } \\
5 \text { (3I.3) for } \\
\text { HoLAP } \\
3 \text { (23) for } \\
\text { Hol-TUIP } \\
\text { I8 (28.I) for } \\
\text { PVP }\end{array}$ \\
\hline Liu et al $2020^{19}$ & $>80$ & $\begin{array}{l}\text { I: } 8(5.3) \\
2: 68(45.4) \\
3: 66(44) \\
4: 8(5.3)\end{array}$ & $44(29.3)$ & $33(22)$ & $65(43.3)$ & $5(3.3)$ & $92(61.4)$ \\
\hline $\begin{array}{l}\text { Hou et al } \\
2016^{18}\end{array}$ & $>80$ & N/A & $\mathrm{N} / \mathrm{A}$ & & $N / A$ & & \\
\hline
\end{tabular}


Table 2 (Continued).

\begin{tabular}{|c|c|c|c|c|c|c|c|c|c|}
\hline \multirow{2}{*}{$\begin{array}{l}\text { Author and } \\
\text { Year }\end{array}$} & \multirow{2}{*}{$\begin{array}{l}\text { Age Groups } \\
\text { (Years) }\end{array}$} & \multirow{2}{*}{$\begin{array}{l}\text { ASA Score, } \\
n(\%)\end{array}$} & \multirow{2}{*}{$\begin{array}{l}\text { APIAC } \\
\text { Therapy, n(\%) }\end{array}$} & \multicolumn{6}{|c|}{ Comorbidities } \\
\hline & & & & DM, n(\%) & HT, & & ND & $n(\%)$ & CVD, $n(\%)$ \\
\hline \multirow{2}{*}{$\begin{array}{l}\text { Ánan et al } \\
2021^{22}\end{array}$} & $\geq 75$ & \multirow[t]{2}{*}{$\mathrm{N} / \mathrm{A}$} & $13(21)$ & \multicolumn{2}{|l|}{$16(42)$} & $25(66)$ & \multicolumn{2}{|c|}{$\mathrm{N} / \mathrm{A}$} & $9(24)$ \\
\hline & $<75$ & & $13(34)$ & \multicolumn{2}{|l|}{$15(24)$} & $31(50)$ & \multicolumn{2}{|c|}{ N/A } & $5(8)$ \\
\hline \multirow{2}{*}{$\begin{array}{l}\text { Majumdar et al } \\
2014^{24}\end{array}$} & $<75$ & \multirow[t]{2}{*}{$\mathrm{N} / \mathrm{A}$} & $53(35.1)$ & \multicolumn{2}{|l|}{$27(17.6)$} & $49(32)$ & \multicolumn{2}{|c|}{$7(4.6)$} & $30(19.6)$ \\
\hline & $\geq 75$ & & $30(61.2)$ & $8(16.3)$ & & $22(44.9)$ & \multicolumn{2}{|c|}{$4(8.2)$} & $17(34.7)$ \\
\hline \multirow[t]{2}{*}{ Gu et al $2012^{23}$} & $<70$ & $2.3 \pm 0.6$ & $34(36.6)$ & \multirow{2}{*}{\multicolumn{6}{|c|}{$N / A$}} \\
\hline & $\geq 70$ & $2.4 \pm 0.7$ & $19(26.8)$ & & & & & & \\
\hline $\begin{array}{l}\text { Bouhadana et al } \\
2021^{13}\end{array}$ & $\geq 75$ & $N / A$ & $N / A$ & $\begin{array}{l}4223(24.1) \\
\text { for LP } \\
2970(24.2) \\
\text { for TURP }\end{array}$ & $\begin{array}{l}\text { II,90I (67.8) } \\
\text { for LP } \\
8475(69.1)\end{array}$ & & V/A & $\begin{array}{l}8775 \\
6085 \\
\text { TUR }\end{array}$ & $\begin{array}{l}\text { (50) for LP } \\
(49.6) \text { for }\end{array}$ \\
\hline
\end{tabular}

Note: *Mean \pm SD.

Abbreviations: HoLEP, Holmium laser enucleation of the prostate; HoLAP, Holmium laser ablation of the prostate; Hol-TUIP, Holmium laser transurethral incision of the prostate; PVP, Photoselective vaporization of the prostate; LP, Laser Prostatectomy; TURP, Transurethral resection of the prostate; ASA, American Society of Anesthesiology; AP/AC, antiplatelet/anticoagulation; DM, diabetes mellitus; HT, hypertension; ND, neurological diseases; CVD, cardiovascular diseases; N/A, not applicable.

Piao et al reported no significant difference between age groups (50-59, 60-69, 70-79 and $\geq 80$ years) in terms of intraoperative, immediate postoperative (recatheterisation, blood transfusion, urinary tract infection) and late postoperative complications (urethral stricture and bladder-neck contracture). They found no significant difference between age groups in terms of transient stress UI $(p=0.180) .{ }^{6}$ Similarly, Mmeje et al reported no significant difference in 30-day complications in Clavien- Dindo grades among groups aged 50-59, 60-69, 70-79, and 80 years. ${ }^{20}$ Continence rates were similar between age groups at their 1-year follow-up (100, 95, 93 and $88 \%$ for groups, respectively; $\mathrm{P}=0.192)$. Tamalunas et al reported no significant difference between age groups $(<70,70$ $79, \geq 80$ years) in perioperative complications- ClavienDindo grade $\geq$ II- (indwelling suprapubic catheter, persistent haematuria, urethral flap, right ureteral ostium injury, aspiration pneumonia) $(\mathrm{p}=0.176) .{ }^{21}$ On the other hand, Gild et al reported that patients aged $\geq 80$ suffered Clavien- Dindo grade $\geq 3 \mathrm{~b}$ complications more frequently (requiring surgical, endoscopic or radiological intervention under general anaesthesia) than their other age groups ( $\leq 60,60-69,70-79, \geq 80$ years), as well as a significant difference between groups in ClavienDindo grade $\geq 3 \mathrm{~b}$ complications $(1.5,1.9,3.9$ and $7.1 \%$; respectively, $\mathrm{p}=0.02){ }^{17}$

\section{Discussion}

\section{Urinary Outcomes}

In recent years, LEP has come to the fore as one of the most popular surgical treatments for BPH. ${ }^{25}$ HoLEP in particular, a minimally-invasive alternative to TURP and open prostatectomy, has contributed substantially to LEP's worldwide popularity. ${ }^{26,27}$ The studies included in this review support the efficacy and safety of HoLEP. Moreover, different surgical approaches using holmium laser have also been shown to improve voiding function in elderly patient groups. Elshal et al showed that HoLEP, HoLAP and Hol-TUIP all enable good functional outcomes in elderly patients. ${ }^{16}$ Both the Holmium laser and other laser types such as Thulium or Greenlight improved functional urinary parameters compared to baseline in elderly patients. Noteworthy, studies show improved functional outcomes at $1,{ }^{21} 3,{ }^{6,19} 6,{ }^{6,22}$ and 12 months ${ }^{14,16,19,20}$ follow-up. Although these follow-up periods are relatively short, laser enucleation of the prostate fulfills the functional expectations of elderly patients.

One of the most challenging potential complications after LEP surgery is UI. Krambeck et al included 1000 patients who underwent HoLEP and reported the postoperative incontinence rate $<5 \% .{ }^{28}$ Elderly patients may have weaker sphincteric tissue than younger patients, which may lead to greater susceptibility to damage caused 
Table 3 Age Groups and Complications According to Clavien-Dindo Classification of the Patients Included in the Study

\begin{tabular}{|c|c|c|c|c|c|c|}
\hline \multirow[t]{3}{*}{ Author and Year } & \multirow[t]{3}{*}{ Age Groups (Years) } & \multicolumn{5}{|c|}{ Complications } \\
\hline & & \multicolumn{5}{|c|}{ Clavien-Dindo Classification, n(\%) } \\
\hline & & $\mathbf{I}$ & II & III & IV & $\mathbf{v}$ \\
\hline \multirow[t]{2}{*}{ Castellani et al $2019^{14}$} & $\geq 75$ & $8(6.2)$ & $4(3.1)$ & $\mathrm{I}(0.8)$ & $\mathrm{I}(0.8)$ & 0 \\
\hline & $<75$ & $30(10.6)$ & $7(2.5)$ & $4(1.5)$ & $2(0.7)$ & 0 \\
\hline \multirow[t]{4}{*}{ Piao et al $2016^{6}$} & $50-59$ & 2 & 2 & 0 & 0 & 0 \\
\hline & $60-69$ & 7 & 21 & 4 & 0 & 0 \\
\hline & $70-79$ & 15 & 17 & 3 & 0 & 0 \\
\hline & $\geq 80$ & 4 & 1 & 0 & 0 & 0 \\
\hline \multirow[t]{3}{*}{ Tamalunas et al $2020^{21}$} & $<70$ & 0 & 0 & $5(2.4)$ & 0 & 0 \\
\hline & $70-79$ & $4(2.4)$ & $\mathrm{I}(0.6)$ & $4(2.4)$ & I (0.6) & 0 \\
\hline & $\geq 80$ & 0 & 0 & $5(4.3)$ & 0 & 0 \\
\hline \multirow[t]{4}{*}{ Mmeje et al $2013^{20}$} & $50-59$ & $3(15)$ & I (5) & 0 & 0 & 0 \\
\hline & $60-69$ & $9(10)$ & $8(8.9)$ & $5(5.6)$ & 0 & 0 \\
\hline & $70-79$ & $12(7.8)$ & $13(8.5)$ & $6(3.9)$ & 0 & 0 \\
\hline & $\geq 80$ & $3(6.7)$ & $6(13.3)$ & $2(4.4)$ & 0 & 0 \\
\hline \multirow[t]{4}{*}{ Gild et al $2020^{17}$} & $\leq 60$ & $N / A$ & $N / A$ & \multicolumn{3}{|c|}{$\geq$ IIlb: 2 (I.5) } \\
\hline & $60-69$ & N/A & $\mathrm{N} / \mathrm{A}$ & \multicolumn{3}{|c|}{$\geq$ IIllb: 12 (1.9) } \\
\hline & $70-79$ & $N / A$ & $N / A$ & \multicolumn{3}{|c|}{$\geq$ IIlb: 34 (3.9) } \\
\hline & $\geq 80$ & $N / A$ & $N / A$ & \multicolumn{3}{|c|}{$\geq$ IIlb: I3 (7.I) } \\
\hline Elshal et al $2013^{16}$ & $\geq 80$ & 4 & 52 & 10 & 2 & 0 \\
\hline Liu et al $2020^{19}$ & $>80$ & \multicolumn{5}{|c|}{$\mathrm{N} / \mathrm{A}$} \\
\hline Hou et al $2016^{18}$ & $>80$ & \multicolumn{5}{|c|}{$N / A$} \\
\hline \multirow[t]{2}{*}{ Ánan et al $2021^{22}$} & $\geq 75$ & \multirow{2}{*}{\multicolumn{5}{|c|}{$N / A$}} \\
\hline & $<75$ & & & & & \\
\hline \multirow[t]{2}{*}{ Majumdar et al $2014^{24}$} & $<75$ & & 27) & $4(2.6)$ & \multirow{2}{*}{\multicolumn{2}{|c|}{ N/A }} \\
\hline & $\geq 75$ & & $0.6)$ & I (2) & & \\
\hline \multirow[t]{2}{*}{ Gu et al $2012^{23}$} & $<70$ & \multirow{2}{*}{\multicolumn{5}{|c|}{$\mathrm{N} / \mathrm{A}$}} \\
\hline & $\geq 70$ & & & & & \\
\hline Bouhadana et al $2021^{15}$ & $\geq 75$ & \multicolumn{5}{|c|}{ N/A } \\
\hline
\end{tabular}

Abbreviation: N/A, not applicable.

by straining the tissue. In addition, an overactive bladder (39-61\%) and detrusor instability (48\%) are common in elderly patients. ${ }^{29}$ Studies on the effect of age on postoperative incontinence after HoLEP have been inconsistent. Houssin et al showed that age was significantly associated with UI after HoLEP. ${ }^{29}$ But in another study, Elmansy et al showed that age is not associated with postoperative stress incontinence after HoLEP. ${ }^{30}$ The studies we reviewed reveal no significant difference in postoperative UI between age groups. Note that as most of the 
studies we reviewed provide no preoperative UI rates that can cause bias. Liu et al reported that no UI was observed in any patient in the 3rd month aged $>80$ years after Greenlight PVP. ${ }^{19}$ Similarly, Gu et al found no difference in postoperative UI after PVP between age groups $<70$ and $\geq 70 .{ }^{23}$ However, Mmeje et al noted that men aged $\geq 80$ years suffered from the highest rates of incontinence (12\%) at the 1-year follow-up after HoLEP. Furthermore, they reported that patients aged $>80$ years presented a $20 \%$ rate of urinary leakage before HoLEP. The difference between these two studies may be due to the different laser types. Another possible explanation is that the higher rate of incontinence after HoLEP in elderly patients may be due to the age-related, radical removal of enlarged prostate glands, which may have adapted and served as part of the men's continence mechanisms.

\section{QoL}

LUTS associated with BPH have a negative impact on the QoL of patients. ${ }^{31}$ The studies we included show that the postoperative QoL scores of patients rose significantly compared to baseline, but also no significant difference between age groups in terms of QoL scores. The studies indicate that laser enucleation of the prostate increases the QoL of patients over 75 and 80 years of age, with no significant difference from younger patient groups. ${ }^{6,14,16,19,21,22}$ Only three studies failed to provide data on postoperative QoL changes in patients. ${ }^{17,18,20}$ Note that most of the studies failed to mention which QoL scale they had used. Only one study used King's Health Questionnaire (KHQ scores). ${ }^{22}$ Nevertheless, according to the studies' outcomes, LEP preserves QoL in elderly patients.

\section{Complications}

The two main challenges in elderly patients undergoing surgery are firstly, the acute morbidity events associated with the procedure and, secondly, maintaining quality of life (QOL) without chronic morbidity. ${ }^{16}$ Several studies provide evidence for the safety and low perioperative morbidity of laser enucleation of the prostate. ${ }^{32,33}$

We found that in those studies that assessed complications according to age groups, they observed no significant differences between age groups. ${ }^{6,14,20-22}$ However, Gild et al found that Clavien- Dindo Grade $>3$ b complications were significantly higher in the $\geq 80$-years patient group than in younger age groups. ${ }^{17}$ The $8.2 \%$ perioperative blood transfusion rate of patients aged $>80$ was significantly higher than those in other age groups $(1.3 \%$ vs $2.7 \%$ vs $8.2 \%$; $<<$
0.001). ${ }^{17}$ We emphasise that unlike other studies, Gild et al did not classify ASA scores or AC/AP therapy according to age. We have no information on ASA scores or AC/AP therapy rates in their elderly group. Their elderly group's ASA scores and AC/AP therapy rates may have been higher, which could have caused the significantly higher incidence of complications in that elderly group. On the other hand, although there was no difference in $\mathrm{AC}$ use between age groups $<70$ and $\geq 70$; $\mathrm{Gu}$ et al found that postoperative haematuria was more common in the $\geq 70$ age group.

The other studies we reviewed did not compare complication rates among groups; they only provided the complications and/or rates in men aged $\geq 80$ years. $^{16,18,19}$ Whereas Elshal et al reported 19.6\% perioperative complications in patients $\geq 80$ years, Liu et al reported shortand long-term postoperative complication rates were $29.8 \%$, and $13.3 \%$, respectively, in the same age group. According to studies investigating patients over the age of 80 , the low rates of perioperative and postoperative complications they report are encouraging.

\section{Comorbidities}

It is not surprising that older men reveal higher ASA scores. Studies in this review reported an ASA score of 2 and above in patients aged $>75$ and $>80$ years. This fact is evidence that much older patients carry a substantial risk, as well as the importance of choosing a safe surgical method. Moreover, AC/AP treatment is common in the $\mathrm{BPH}$ age group. AC/AP treatment may cause significant complications in older patients undergoing surgery. Studies show that LEP in elderly patients taking AP/AC is as safe as in younger populations. Elshal et al showed that ongoing anticoagulation therapy in the patient group $>80$ years old is not an independent predictor of perioperative morbidity in patients undergoing HoLEP (OR, 1.03; 95\% CI, $1.07-3.9 ; \mathrm{P}=0.2) .{ }^{16}$ Similarly, Liu et al detected no significant difference in postoperative haematuria in patients $>80$ years with and without oral anticoagulant use who underwent PVP $(9.1 \%$ vs $6.6 \%$, $\mathrm{p}=0.6){ }^{19}$ However, those studies (see Complications) report a difference in perioperative complications.This difference observed among the same age groups is attributable to the fact that Lui et al study patients had ASA scores 3 and higher, and suffered more often from cardiovascular diseases. ${ }^{16,19}$ Gild et al found that taking lowdose acetylsalicylic acid (OR: 1.5, 95\%; CI:1.0-2.2, $\mathrm{p}=0.015$ ), or oral anticoagulation (OR: 2.3 , 95\% CI: $1.2-$ 4.0, $\mathrm{p}=0.022$ ), as well as low-molecular-weight heparin 
(OR: 2.2, 95\% CI: 1.4-3.6, p=0.037) were independently associated with prolonged catheterisation in patients who underwent HoLEP. ${ }^{17}$ Such differences between studies may be due to their diverging pre- and post-surgical AC/ AP regimens.

Various age groups have different impacts on the surgical outcomes, perioperative complications and safety. The main reasons can be assumed as higher ASA score, various comorbidities, use of AC/AP, advanced age-related sphincteric insufficiency and therefore the risk of perioperative and postoperative complications are common. In general, it is expected that the surgical outcome is worse and the risks are higher in elderly patients. However, the studies included in our review revealed that LEP is an effective and safe method in patients of various age groups. To the best of our knowledge, this is the first review focussing on LEP procedures in elderly patients. However, our review has certain limitations. Firstly, most of the studies were of retrospective nature, revealed large differences in patient numbers, varying study designs; all these factors make our data heterogeneous. Secondly, because of the paucity of published studies investigating the effect of laser enucleation of the prostate together with age-stratification, the number of studies included in the review is small.

\section{Conclusion}

Laser enucleation of the prostate due to LUTS is an efficient and safe procedure in elderly patients, resulting in improved functional outcomes, low morbidity, and few perioperative complications. LEP procedures can be also offered to patients over 65 years of age suffering LUTS associated with BPH. Future studies with more patients will enable us to more clearly demonstrate the efficacy and safety of LEP in elderly patients.

\section{Ethics}

This research did not involve human subjects or animals. As this is a review of the literature, no ethics approval was necessary.

\section{Author Contributions}

M.Y., J.E. and A.M. designed the study. M.Y. and J. E. wrote the manuscript. M.Y., J.E., R.S.I., C.G. and A.M. performed the analysis and interpretation of data as well as the manuscript revision. C.G. and A.M. reviewed the manuscript. A.M. supervised the manuscript. All authors made a significant contribution to the work reported, whether that is in the conception, study design, execution, acquisition of data, analysis and interpretation; took part in drafting, revising or critically reviewing the article; gave final approval of the version to be published; have agreed on the journal to which the article has been submitted; and agree to be accountable for all aspects of the work.

\section{Funding}

No extra funding was received.

\section{Disclosure}

Prof. Dr. Christian Gratzke is advisor for Astellas Pharma GmbH, DE, Ipsen Pharma GmbH, DE, Steba Biotech S.A., LUX, Bayer Pharma, DE, Olympus Winter \& Ibe GmbH, DE, Medi-Tate Ltd., IL, MSD, DE, Astra-Zeneca, UK and Roche, $\mathrm{CH}$. He receives speaker fees from Amgen, USA, Astellas Pharma GmbH, DE, Ipsen Pharma GmbH, DE, JanssenCilag GmbH, BEL, Bayer Pharma, DE, Takeda Pharmaceuticals, JPN and medac GmbH, DE. A. Miernik receives research funding from the German Federal Ministry of Education and Research, Berlin (D). He receives support for his travel activities from the European Society of Urology, Arnhem (NL), and German Society of Urology, Düsseldorf (D). A. Miernik is also a consultant for: KLS Martin, Tuttlingen (D), Avateramedical, Jena (D), LISA LaserProducts GmbH, Katlenburg-Lindau (D), Schoelly fiber optics GmbH, Denzlingen (D), Dornier MedTech Laser GmbH (D), Medi-Tate Ltd. (IL, USA) and B. Braun New Ventures $\mathrm{GmbH}$, Freiburg (D). A. Miernik is speaker for the companies Richard Wolf GmbH (D) and Boston Scientific (USA). He also served as a reviewer for the Ludwig Boltzmann Gesellschaft, Wien (A). In addition, Prof. Dr. Arkadiusz Miernik has a patent WO2014173468A1 licensed to Purenum $\mathrm{GmbH}$, German start-up, a patent EP2988681A1 licensed to Purenum GmbH, German start-up, a patent WO2015082372A1 pending; a patent WO2017207361A1 pending; a patent DE102019220537A1 pending; a patent DE102019134352A1 pending; a patent DE102015003560B3 issued; a patent WO2021005060A1 pending. M. Yilmaz, J. Esser and Rodrigo Suarez have no conflicts of interest to declare.

\section{References}

1. Gratzke C, Bachmann A, Descazeaud A, et al. EAU guidelines on the assessment of non-neurogenic male lower urinary tract symptoms including benign prostatic obstruction. Eur Urol. 2015;67 (6):1099-1109. doi:10.1016/j.eururo.2014.12.038 
2. Lee SWH, Chan EMC, Lai YK. The global burden of lower urinary tract symptoms suggestive of benign prostatic hyperplasia: a systematic review and meta-analysis. Sci Rep. 2017;7(1):7984. doi:10.1038/ s41598-017-06628-8

3. Oelke M, Bachmann A, Descazeaud A, et al. EAU guidelines on the treatment and follow-up of non-neurogenic male lower urinary tract symptoms including benign prostatic obstruction. Eur Urol. 2013;64 (1):118-140. doi:10.1016/j.eururo.2013.03.004

4. Reich O, Gratzke C, Bachmann A, et al. Morbidity, mortality and early outcome of transurethral resection of the prostate: a prospective multicenter evaluation of 10,654 patients. $J$ Urol. 2008;180 (1):246-249. doi:10.1016/j.juro.2008.03.058

5. Shah AA, Gahan JC, Sorokin I. Comparison of robot-assisted versus open simple prostatectomy for benign prostatic hyperplasia. Curr Urol Rep. 2018;19(9):71. doi:10.1007/s11934-018-0820-1

6. Piao S, Choo MS, Kim M, Jeon HJ, Oh SJ. Holmium laser enucleation of the prostate is safe for patients above 80 years: a prospective study. Int Neurourol J. 2016;20(2):143-150. doi:10.5213/ inj.1630478.239

7. Nair SM, Pimentel MA, Gilling PJ. A review of laser treatment for symptomatic BPH (Benign Prostatic Hyperplasia). Curr Urol Rep. 2016;17(6):45. doi:10.1007/s11934-016-0603-5

8. Rieken M, Ebinger Mundorff N, Bonkat G, Wyler S, Bachmann A. Complications of laser prostatectomy: a review of recent data. World J Urol. 2010;28(1):53-62. doi:10.1007/s00345-009-0504-z

9. Xiao KW, Zhou L, He Q, et al. Enucleation of the prostate for benign prostatic hyperplasia thulium laser versus holmium laser: a systematic review and meta-analysis. Lasers Med Sci. 2019;34 (4):815-826. doi:10.1007/s10103-018-02697-x

10. Vincent MW, Gilling PJ. HoLEP has come of age. World J Urol. 2015;33(4):487-493. doi:10.1007/s00345-014-1443-X

11. Alkan I, Ozveri H, Akin Y, Ipekci T, Alican Y. Holmium laser enucleation of the prostate: surgical, functional, and quality-of-life outcomes upon extended follow-up. Int Braz J Urol. 2016;42 (2):293-301. doi:10.1590/s1677-5538.ibju.2014.0561

12. Sun I, Yoo S, Park J, et al. Quality of life after photo-selective vaporization and holmium-laser enucleation of the prostate: 5-year outcomes. Sci Rep. 2019;9(1):8261. doi:10.1038/s41598-019-44686-2

13. Liberati A, Altman DG, Tetzlaff J, et al. The PRISMA statement for reporting systematic reviews and meta-analyses of studies that evaluate health care interventions: explanation and elaboration. PLoS Med. 2009;6(7):e1000100. doi:10.1371/journal.pmed.1000100

14. Castellani D, Pirola GM, Gasparri L, et al. Are outcomes of thulium laser enucleation of the prostate different in men aged 75 and over? A propensity score analysis. Urology. 2019;132:170-176. doi:10.1016/j.urology.2019.06.025

15. Bouhadana D, Nguyen DD, Zhang X, et al. Safety and efficacy of TURP vs. laser prostatectomy for the treatment of benign prostatic hyperplasia in multi-morbid and elderly individuals aged $\geq 75$. World J Urol. 2021. doi:10.1007/s00345-021-03779-2

16. Elshal AM, Elmansy HM, Elhilali MM. Transurethral laser surgery for benign prostate hyperplasia in octogenarians: safety and outcomes. Urology. 2013;81(3):634-639. doi:10.1016/j. urology.2012.11.042

17. Gild P, Lenke L, Pompe RS, et al. Assessing the outcome of holmium laser enucleation of the prostate by age, prostate volume, and a history of blood thinning agents: report from a single-center series of $>1800$ consecutive cases. $J$ Endourol. 2021;35:639-646. doi:10.1089/end.2020.0605
18. Hou C-M, Chen C-L, Lin Y-H, Yang P-S, Chang P-L, Tsui K-H. Treatment outcomes of benign prostate hyperplasia by thulium vapoenucleation of the prostate in aging men. Urol Sci. 2016;27 (4):230-233. doi:10.1016/j.urols.2016.03.006

19. Liu X, Yuan F, Xue Md B. GreenLight XPS 180-W laser vaporization of prostate in high-risk elderly patients: a single-center experience. Photobiomodul Photomed Laser Surg. 2020;38(6):380-384. doi:10.1089/photob.2019.4735

20. Mmeje CO, Nunez-Nateras R, Warner JN, Humphreys MR. Agestratified outcomes of holmium laser enucleation of the prostate. BJU Int. 2013;112(7):982-989. doi:10.1111/bju.12063

21. Tamalunas A, Westhofen T, Schott M, et al. The clinical value of holmium laser enucleation of the prostate in octogenarians. Low Urin Tract Symptoms. 2021;13(2):279-285. doi:10.1111/luts.12366

22. Anan G, Iwamura H, Mikami J, et al. Efficacy and safety of holmium laser enucleation of the prostate for elderly patients: surgical outcomes and King's Health Questionnaire. Transl Androl Urol. 2021;10 (2):775-784. doi:10.21037/tau-20-1309

23. Gu X, Strom K, Spaliviero M, Wong C. Does age affect the efficacy and safety of GreenLight HPS laser photoselective vaporization prostatectomy? Aging Male. 2012;15(1):63-67. doi:10.3109/ 13685538.2011.632452

24. Majumdar R, Mirheydar HS, Palazzi KL, Lakin CM, Albo ME, Parsons JK. Prostate laser vaporization is safe and effective in elderly men. Urol Ann. 2015;7(1):36-40. doi:10.4103/0974-7796.148595

25. Herrmann TRW, Gravas S, de la Rosette JJ, Wolters M, Anastasiadis AG, Giannakis I. Lasers in transurethral enucleation of the prostate-do we really need them. J Clin Med. 2020;9(5):1412. doi: $10.3390 /$ jcm 9051412

26. Elzayat EA, Habib EI, Elhilali MM. Holmium laser enucleation of the prostate: a size-independent new "gold standard". Urology. 2005;66(5 Suppl):108-113. doi:10.1016/j.urology.2005.06.006

27. Kuntz RM, Lehrich K, Ahyai SA. Holmium laser enucleation of the prostate versus open prostatectomy for prostates greater than 100 grams: 5-year follow-up results of a randomised clinical trial. Eur Urol. 2008;53(1):160-166. doi:10.1016/j.eururo.2007.08.036

28. Krambeck AE, Handa SE, Lingeman JE. Experience with more than 1000 holmium laser prostate enucleations for benign prostatic hyperplasia. J Urol. 2013;189(1Suppl):S141-145. doi:10.1016/j. juro.2012.11.027

29. Houssin V, Olivier J, Brenier M, et al. Predictive factors of urinary incontinence after holmium laser enucleation of the prostate: a multicentric evaluation. World J Urol. 2021;39(1):143-148. doi:10.1007/s00345-020-03169-0

30. Elmansy HM, Kotb A, Elhilali MM. Is there a way to predict stress urinary incontinence after holmium laser enucleation of the prostate? J Urol. 2011;186(5):1977-1981. doi:10.1016/j.juro.2011.06.063

31. Giuliano F. Lower urinary tract symptoms and sexual dysfunction: a common approach. BJU Int. 2008;101(Suppl 3):22-26. doi:10.1111/j.1464-410X.2008.07494.x

32. Shah HN, Mahajan AP, Hegde SS, Bansal MB. Peri-operative complications of holmium laser enucleation of the prostate: experience in the first 280 patients, and a review of literature. BJU Int. 2007;100 (1):94-101. doi:10.1111/j.1464-410X.2007.06867.x

33. Vavassori I, Valenti S, Naspro R, et al. Three-year outcome following holmium laser enucleation of the prostate combined with mechanical morcellation in 330 consecutive patients. Eur Urol. 2008;53 (3):599-604. doi:10.1016/j.eururo.2007.10.059 


\section{Publish your work in this journal}

Clinical Interventions in Aging is an international, peer-reviewed journal focusing on evidence-based reports on the value or lack thereof of treatments intended to prevent or delay the onset of maladaptive correlates of aging in human beings. This journal is indexed on PubMed Central, MedLine, CAS, Scopus and the Elsevie
Bibliographic databases. The manuscript management system is completely online and includes a very quick and fair peer-review system, which is all easy to use. Visit http://www.dovepress.com/ testimonials.php to read real quotes from published authors. 